\title{
EFFECT OF CORE AND TREADMILL TRAININGS ON SKELETAL MIN- ERALIZATION IN POST-MENOPAUSAL OSTEOPOROTIC WOMEN
}

\section{Mohamed Mostafa M. Essa ${ }^{1}$, Hossam El-Din Fawaz ${ }^{2}$, Hamada Ahmed Hamada ${ }^{2}$,} Mariam Abd-Elmoneem Ameer *2, Salam Mohamed Elhafez ${ }^{2}$.

${ }^{1}$ Department of Biomechanics, Faculty of Physical Therapy, Musr University, Giza, Egypt.

${ }^{2}$ Department of Biomechanics, Faculty of Physical Therapy, Cairo University, Giza, Egypt.

\section{ABSTRACT}

Background: Osteoporosis is considered as the main cause of bone weakness and fracture in old age. The main purpose of this study was to explore the effect of core and treadmill trainings on skeletal mineralization in postmenopausal osteoporotic women.

Materials and Methods: Twenty osteoporotic postmenopausal women ageing between 50 to 60 years with BMI between 20.2 to $24.9 \mathrm{~kg} / \mathrm{m}^{2}$ engaged in this study. They were assigned randomly into two equal groups. Participants of the group (I) received core training programme, while the group (II) received treadmill training programme lasting three months period. Bone mineral densities (BMD) at L2-L4 and right femoral neck were measured using a dual energy X-ray absorptiometry (DEXA). The participants were tested twice; before and after the training programmes.

Results: The statistical analysis showed that there was a significant increase in femoral neck bone mineral density in group (II) in the post-treatment condition $(p<0.05)$. Moreover, there was a significant improvement $(p<0.05)$ in femoral neck BMD in group (II) compared with group (I). However, there were no significant differences in the lumber spine bone mineral density between the pre-treatment and post-treatment conditions for either group $(p>0.05)$.

Conclusions: It can be concluded that the treadmill training programme is an effective treatment policy to improve BMD (especially at hip region) and help in decreasing bone loss in postmenopausal women.

KEY WORDS: Bone mineral density, Core Training, Treadmill Training, Aging, Osteoporosis.

Address for correspondence: Mariam Abd-Elmoneem Ameer, Department of Biomechanics, Faculty of Physical Therapy, Cairo University, Giza, Egypt. Tel: +201094169696

E-Mail: mariam_ameer7@hotmail.com

\begin{tabular}{|l|l|}
\hline \multicolumn{3}{|c|}{ Access this Article online } \\
\hline Quick Response code & \multicolumn{1}{|c|}{ International Journal of Physiotherapy and Research } \\
ISSN 2321- 1822
\end{tabular}

\section{INTRODUCTION}

Osteoporosis is a degenerative systemic bony disease with low bone mass and micro-architectural deterioration [1]. About $6 \%$ of men and $21 \%$ of women aged between $50-84$ years in Europe and North America are classified as osteoporotic. Osteoporosis is one of the main cause of increasing bone weakness, and hip fractures secondary to trauma [2]. The osteoporotic fracture occurs with low bone mineral density
(BMD), so prohibition of age-related bone loss is an important point in the research [3].

Adaptability of bone tissue is achieved through a continuous change in response to compulsory mechanical stress. The bone remodeling occurs through interaction between the osteoclasts and osteoblasts. Osteocalasts cause degeneration of bone cells while the osteoblasts deposit new bone cells. Acceleration of bone deposition will 
normally occur with increasing stresses, through dynamic remodeling activity in response to stress changes [4]. In the third decade of life, maximum bone mineral density usually attained and maintained for about another 10 years through the balance between osteoclastic and osteoblastic activities. Be-yond the 30 to 40 years old age, failure of the osteoblastic action to com-pletely balance the osteoclastic activity leading to an increased size of the resorption cavities and decreased bone mass [4].

Concurrent age-related declines of muscular force and bone loss have been stated in postmenopausal women. The maximum muscle force should be taken into consideration as a strong factor which affects the mechanical characteristics of bone [5].

Muscles about the abdominal and lumbar regions are core muscles, such as erector spinae, rectus abdominis, external oblique, quadratus lumborum, and gluteus medius [1]. The core programme should be planned and progressed gradually, as exercising the core musculature is more than trunk strengthening. Correction of muscle imbalance through recuperation of normal muscle mobility and length is the start of core programme. Such as tightness and overactivity of the main hip flexor (iliopsoas muscle) may lead to reciprocal inhibition of the primary hip extensor (gluteus maximus muscle) [6].

Regarding the relation-ships between BMD and muscle strength, Sinaki et al. [5] found a decrease in both elbow flexors strength and BMD of the radius with increasing age. However, a positive correlation between back exten-sor strength and L2-L4 BMD was detected. Also, Zimmermann et al. [7] found that the hip flexors peak torque is significantly correlated to the lumbar vertebrae and hip BMD in postmenopausal women There is lack of comparisons between different types of exercises effect on in-creasing the bone mass, particularly in elder women [8]. Recent meta-analyses of exercise studies showed positive effects of physical exercise on BMD in postmenopausal women. But it is uncertain, whether the most effective strategy which can regain or maintain BMD has yet been found [3]. Howe et al. [9] reported that the different exercise programs which comprised static weight-bearing exercises, low or high force dynamic weight bearing exercises, and low or high force non-weight bearing exercises were the most effective programs for the spine BMD.

To our knowledge, no studies have used core training programmes in patients with osteoporosis, and most studies have focused on treadmill exercises and examined women with normal or low BMD. So, the current study is performed to explore the effect of core and treadmill training programmes on skeletal mineralization of osteoporotic postmenopausal women. It might provide physiotherapists, clinicians and researchers the best type of training that enhances the BMD of osteoporotic postmenopausal women. In turn, it might help in reduction of the clinical consequences and economic loss suffered by millions of osteoporotic patients.

\section{MATERIALS AND METHODS}

Subjects: Twenty postmenopausal osteoporotic women (age between 50 and 60 years) with body mass index (BMI) between 20.2 and $24.9 \mathrm{~kg} / \mathrm{m}^{2}$ participated in this study. They were with at least one year postmenopause and T-score of BMD (2.5 SD or more below the young adult mean). Patients with history of musculoskeletal problems, severe renal, hepatic, endocrine, cardiac impairments and uncontrolled hypertension or diabetes were excluded. They were randomly distributed into two groups; first experimental "group I, n=10" and second experimental "group II, $n=10$ ". Before data collection, each participant signed consent form that approved by Research Ethical Committee, Faculty of Physical Therapy, Cairo University (No: P.T.REC/012/ 00469).

Instruments: Dual energy x-ray absorptiometry (DEXA) using Hologic QDR-4500 (Hologic, Bedford, MA, USA) was used to measure the lumbar spine (L2-L4) and right femoral neck BMD (in grams per square centimeter). Treadmill (Quinton Fitness Equipment, Clubtrack, Fontana, $C A$, ) was used in the current study for the treadmill training programme.

Procedure: A brief explanation session about the nature, aims and the tests of the study was accomplished to each participant. The assessment and treatment procedures were clear for all participants. Before performing the testing procedures, the data (age, weight, height, and 
calculation of BMI) were recorded for each subject. Each participant of both groups in the current study was assessed pre-treatment and post-treatment, after three months period of receiving core and treadmill training programmes. Bone mineral density (BMD) was assessed in the lumbar spine (L2-L4) and right femoral neck by Dual Energy X-ray Absorptiometry using Hologic QDR-4500 (Hologic, Bedford, MA, USA). Consistent procedures in acquiring all scans were followed. Before patients' scanning, it was ensured that patients can bear supine lying position (lying flat on their back) for at least 10 minutes and the scan area is free from all radio-opaque objects. The machines were calibrated daily, as per the manufacturer's recommendations.

For lumbar spine scans, the participant assumed supine lying position on the scanning table. The participants' knees were flexed and shins were elevated by a positioning cube to level the spine against the table and eliminate lumbar lordosis. BMD of L2-L4 in the posterior-anterior projection was measured [10]. The spine was centered in the field of scan and was not rotated. The scan extended up to include the level of lowest vertebra and ribs (the level of T12) and down to the pelvic brim (the level of L4-L5 inter-space) [11]. For femoral neck scans, the participant's leg was straight on the table with $15^{\circ}-25^{\circ}$ of internal rotation. This was done by participant's foot flexion before doing the internal rotation, and then moving the foot to neutral position after the straps was in place. The internal rotation of the limb was confirmed by seeing little or none of lesser trochanter on the scan [11]. The same scan mode was used in the pre- and post-treatment conditions, the scan length and width was kept at the default settings in most situations.

Intervention protocols: Participants of the first experimental group (I) received core training programme three sessions weekly for three months period. Each exercise has 15 repetitions at each session [12] in form of warm-up exercise and progressed core strengthening exercises (curl up, side-bridge and bird dog).

The core training programme began with flexion-extension cycle (cat-camel) motion from quadruped position (5-8 cycles) (Fig. 1) [13].
Each participant performed the beginner's core stability exercise after warming up.

Fig. 1: cat-camel motion from quadruped position.

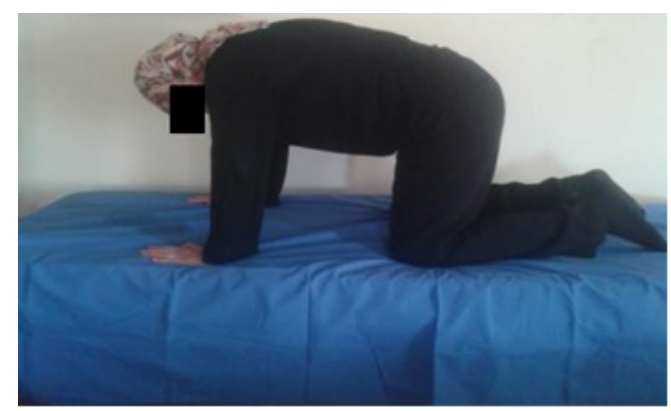

In the curl up exercise, a neutral spine posture was preserved by placing the participant's hands under the lumbar spine. One knee was flexed but the other knee was straight, and then alternates the bent leg (right to left) midway through the repetitions. The participant's head and shoulders were raised off the mat, so the motion was in the thoracic region only (Fig. 2). Raising the elbows off the mat made the exercise more challenging. The position maintained for 7-8 seconds without holding the breath [13].

Fig. 2: curl up exercise with head, shoulder and one knee flexion.

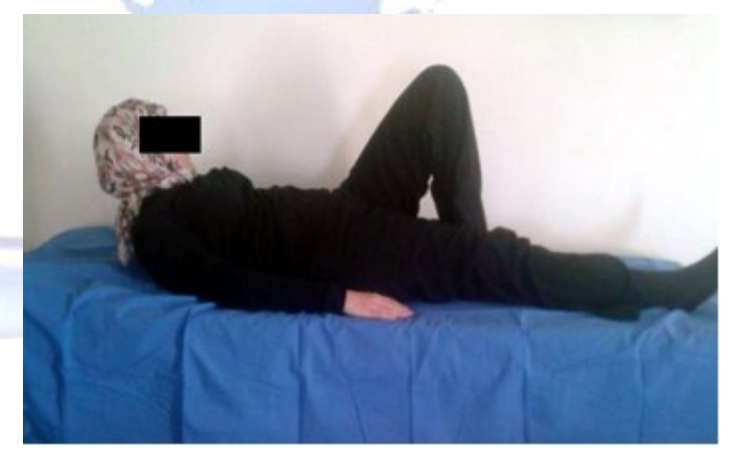

The participant assumed side lying position on the right side in isometric side-bridge exercise. The right shoulder was abducted, so the arm was in vertical alignment with the ground and the forearm was rested on the bed. The pelvis was raised off the mat and held in a straight line "plank" position. This posture was held for 7-8 seconds. The exercise was advanced by putting the upper leg in front of the lower one (Fig. 3). The participant supported the trunk with resting the forearms on the bed, bending the elbows $90^{\circ}$, and resting the toes on the bed. The participant maintained the spine in a mid-position for 7-8 seconds. Instruction was given to the participant to roll and assume prone lying position on elbows and feet for 7-8 seconds. Finally, the participant was instructed to assume side 
lying position on the left side and perform the exercise as illustrated before [13].

Fig. 3: side-bridge exercise with upper limb crossing the lower one.

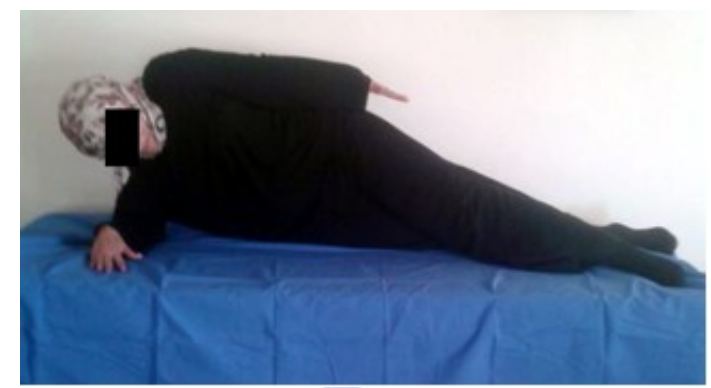

In the bird dog exercise, the participant assumed the quadruped position on hands and knees. The subject raised the right arm and left leg as much as possible to be (opposite lower and upper limbs) in the same line with the trunk with maintaining the normal curve of the lumbar spine (Fig. 4). The participant holds this posture for 7-8 seconds with deep breathing. Lowered the hand and knee to sweep the floor with them and raised again for the next repetition. Then, the participant was asked to alternate sides [13].

Fig. 4: bird dog exercise from quadruped position.

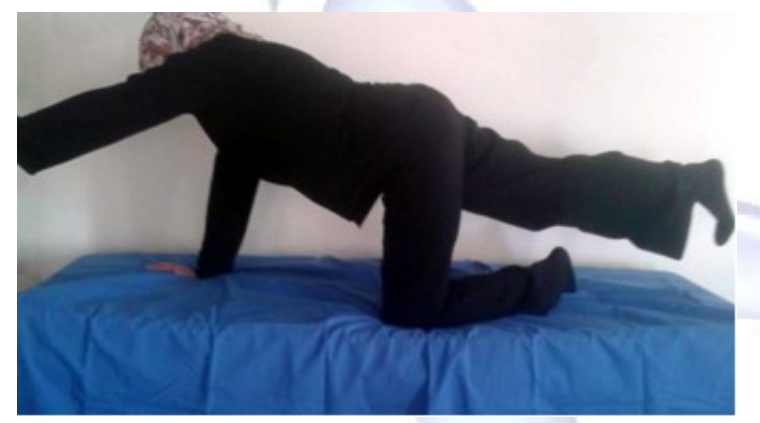

While the participants of the second experimental group (II) received treadmill training programme, three sessions weekly. All treadmill training sessions were performed on a Quinton treadmill (Quinton Fitness Equipment, Clubtrack, Fontana, CA). A familiarization session of 5-10 minutes on the treadmill apparatus was done for the participants prior to the first session. The treadmill without inclination was set for the training period. The training session was started with 5 minute warm-up on the treadmill at $45 \%$ of heart rate reserve (HRR, Karvonen method). After warming up, the effort was increased up to $65 \%$ of the subjects' HRR. Two miles per session was completed at the first week, 2.5 miles per session at the second week, while at the third week three miles per session and the fourth week 3.5 miles per session [14]. The treadmill training programme was conducted for three months period with the same protocol of the first month.

Data analyses: It was proposed to compare between both groups "between-subject effect" for the (L2-L4) BMD, and right femoral neck BMD in each of the "pre-treatment" and "post-treatment" situations. Also, it was intended to compare between the "pre-treatment" and "post-treatment" conditions "within-subject effect" for these variables of interest in both tested groups. Finally, the interaction effect was examined. $2 \times 2$ Mixed design MANOVA was used to determine whether there were significant differences in the set of dependent variables across the two experimental groups which received core training and treadmill training programmes and across the two times that measurements were taken.

\section{RESULTS}

All statistical measures were achieved using the Statistical Package for Social science (SPSS) program version 18 for windows. Before data analysis, data were screened for normality assumption, and homogeneity. This exploration was necessary for parametric calculation of difference analysis. To determine similarity between the groups at base line, participant's age, height, weight, and body mass index (BMI) were compared using independent t-tests.

The current study involved two independent variables. The first one was the tested group which had two levels (group I \& group II). The second one was the measuring periods which had two levels (pre- and post-treatments conditions). The two dependent variables were L2-L4 BMD and Femoral neck BMD. Accordingly, $2 \times 2$ Mixed design MANOVA was used to compare the tested variables of interest at different tested groups and measuring periods. MANOVA was conducted with the initial alpha level set at 0.05 .

Demographic data of patients: As specified by the unpaired t-tests, the mean values of age, weight, height, and BMI between subjects in both groups show no significant differences $(p>0.05)$. The demographic data of the participants are shown in (Table 1). 
Table 1: Descriptive statistics and unpaired t-tests for demographic data of the participants in both groups.

\begin{tabular}{|c|c|c|c|c|}
\hline & $\begin{array}{c}\text { Age } \\
\text { (vears) }\end{array}$ & $\begin{array}{c}\text { Weight } \\
(\mathrm{kg})\end{array}$ & Height (cm) & BMI (kg/m²) \\
\hline Group (I) & $53.5 \pm 2.75$ & $68.6 \pm 8.68$ & $167.8 \pm 6.46$ & $24.31 \pm 1.69$ \\
\hline Group (II) & $52.3 \pm 2.11$ & $68.3 \pm 8.34$ & $169.6 \pm 7.61$ & $23.67 \pm 1.74$ \\
\hline t-value & 1.092 & 0.079 & 0.57 & 0.825 \\
\hline p-value & 0.289 & 0.938 & 0.576 & 0.42 \\
\hline
\end{tabular}

Bone mineral density changes: Concerning the L2-L4 BMD and Femoral neck BMD, the statistical analysis revealed that there was significant within subject effect $(F=6.351, p=0.000)$ and treatment*time effect $(F=4.074, p=0.036)$ but there was no significant between subject effect $(F=1.425, p=0.268)$. Table ( 2 ) represents the mean \pm SD and multiple pairwise comparisons for all dependent variables in both groups in different measuring periods. A significant increase in BMD at the femoral neck (2.3\%) and small positive change at the lumbar spine $(0.22 \%)$ were found in group (II) which received treadmill programme. While in group (I) which received core training programme, small positive changes were found at the femoral neck $(0.36 \%)$ and lumbar spine $(0.23 \%)$.

Multiple pairwise comparison tests shown that there were significant increase of femoral neck BMD in the post-treatment condition compared with the pre-treatment one in group (II) only $(p<0.05)$. Regarding between subject effects, multiple pairwise comparisons revealed that there were significant increase $(p<0.05)$ in femoral neck BMD in group (II) compared with group (I), with no significant differences in L2-L4 BMD between both groups in the pre- and post-treatment conditions.

Table 2: Descriptive statistics and multiple pairwise comparison tests (Post-hoc tests).

\begin{tabular}{|c|c|c|c|c|c|c|}
\hline \multicolumn{4}{|c|}{ Group II (N=10) } & \multicolumn{2}{|c|}{ Group I (N=10) } & \multirow{2}{*}{ Dependent variables } \\
\hline \multicolumn{2}{|c|}{ Post-treatment } & \multicolumn{2}{|c|}{ Pre-treatment } & Post-treatment & Pre-treatment & \\
\hline \multicolumn{2}{|c|}{$0.7944 \pm 0.037$} & \multicolumn{2}{|c|}{$0.7926 \pm 0.032$} & $0.7943 \pm 0.31$ & $0.7924 \pm 0.03$ & L2-L4 BMD (g/ $\left./ \mathrm{cm}^{2}\right)$ \\
\hline \multicolumn{2}{|c|}{$0.577 \pm 0.014$} & \multicolumn{2}{|c|}{$0.564+0.018$} & $0.557 \pm 0.022$ & $0.555 \pm 0.022$ & Femoral neck BMD $\left(\mathrm{g} / \mathrm{cm}^{2)}\right.$ \\
\hline \multicolumn{7}{|c|}{ Multiple pairwise comparisons between pre- and post-treatment values for all dependent variables } \\
\hline \multicolumn{3}{|c|}{ Femoral neck BMD } & \multicolumn{3}{|c|}{ L2-LABMD } & Dependent variables \\
\hline \multicolumn{3}{|c|}{0.534} & \multicolumn{3}{|c|}{0.614} & Group I \\
\hline \multicolumn{3}{|c|}{$0.000^{*}$} & \multicolumn{3}{|c|}{0.632} & Group II \\
\hline \multicolumn{7}{|c|}{ Multiple pairwise comparisons between group (I) and group (II) for all dependent variables } \\
\hline \multicolumn{3}{|c|}{ Post-treatment } & \multicolumn{3}{|c|}{ Pre-treatment } & Measuring periods \\
\hline $\begin{array}{l}\text { Femoral } \\
\text { neck BMD }\end{array}$ & \multicolumn{2}{|c|}{ L2-L4 BMD } & \multicolumn{2}{|c|}{ Femoral neck BMD } & L2-LA BMD & Dependent variables \\
\hline $0.028^{*}$ & \multicolumn{2}{|c|}{0.312} & \multicolumn{2}{|c|}{0.995} & 0.989 & $\begin{array}{l}\text { Group (II) Vs. } \\
\text { group (II) }\end{array}$ \\
\hline
\end{tabular}

\section{DISCUSSION}

The main objective of the current study was to explore the effect of core and treadmill trainings on skeletal mineralization in postmenopausal osteoporotic women. The findings revealed that the treadmill training program can help to increase bone mineral density in postmenopausal women, although these improvements were limited to the hip region (femoral neck). The significant increase of femoral neck BMD in group (II) compared with group (I), indicated that the treadmill training as weight bearing exercise had more effect on BMD than the core training programme as strength training.

In weight bearing activities, the mechanical loads are transferred to the skeleton by gravitational forces and muscle pull where bone cells selectively react to mechanical stresses to decrease and increase BMD [15]. The piezoelectric effect causes deposition of bone at the points of compressional stress. The physical stress stimulates osteoblastic deposition of bone, which causes a negative potential at the compressed site and a positive potential elsewhere in the bone, so BMD increases [16].

The findings of this study come in agreement with the results of a study done by Chow et al. [17] who stated an improvement in BMD with treadmill exercise at $80 \%$ of $\mathrm{HR}_{\max }$. Also, Nelson et al. [18] showed useful effects of walking at $75-80 \%$ of $\mathrm{HR}_{\max }$ for 50 minutes, 3-4 times a week on BMD in postmenopausal women. Chien et al. [19] reported that there is an increase in BMD about $6.8 \%$ in the femur, $p<0.05$, and an increase in BMD about $2 \%$ in lumbar spine (L2-L4), $p>0.05$ for the exercise group which received treadmill, and stepping exercise. This is matched with our results of detecting a significant improvement in $\mathrm{BMD}$ at the femoral neck (by $2.3 \%$ ) which is more than the small positive changes at the lumbar spine (by $0.22 \%$ ) in group (II) which received treadmill program.

Also, agreed with Kerr et al. [20] who reported large bone density at the femoral trochanter (the attachments of the gluteus medius, piriformis, gluteus minimus muscles), the inter trochanter site (insertion of psoas and iliacus muscle) and Ward's triangle but not at the femoral neck among postmenopausal women perform 
strength training. This returned to muscle attachments are found on trochanters and not on femoral neck. So, the transfer of impact forces may not be effective in encouraging bone growth at the femoral neck in group (I) which received core program. Also, the conducted core program just included cat camel motion, curl up, bird dog, and isometric side bridge exercises only.

Bemben et al. [21] established positive correlations among hip strength exercise (flexion, extension, and abduction), leg strength exercise (hamstring curls, and leg press), and hip BMD sites (femoral neck, trochanter, Ward's triangle, and total hip). So, exercises depending on gravitational forces or ground reaction forces are most effective in enhancing bone remodeling in the femoral neck.

The results of the current study disagreed with Korpelainen et al. [22] who found no increase in BMD in women who treated with weight bearing exercise program consisted of stair climbing, walking, dancing, knee bends, stamping, leg lifts, heel rise, and stepping up and down from benches. While walking in combination with strengthening for back, legs, arms, core and aerobic training have demonstrated a small positive effect on hip BMD $(p=0.066)$ in postmenopausal women, with a treatment effect of $1 \%$ after 12 months of training [23].

Regarding L2-L4 BMD, our study shown that there were no significant differences between both groups which received core and treadmill programs. This result contradicts the finding of Shanb et al. [24] who suggested that there is a significant increase of the lumbar spine BMD after either weight bearing- or non-weight bearing exercise programs because of strong osteogenic stimulus during muscular contraction. Also, kohrt et al. [8] agreed with results of the current study in noting a significant increase in the femoral neck BMD with the ground reaction force group only (treadmill group), and contradicted with our findings in reporting the significant increases in lumbar spine BMD with both ground reaction force (treadmill group), and joint reaction force groups (exercise group).

Bone remodeling can take four to six months or more. Lester et al. [25] stated that training interventions of longer durations (6-36 months) have reported positive BMD increases whereas those of shorter durations (less than 6 months) have failed to show similar findings. So, it may be too earlier to detect the effects of training programs after three months period. The findings of our study cannot be used for all postmenopausal women, as ageing alters the response of bone to mechanical loading which may affect the response to the treadmill and core programs.

\section{CONCLUSION}

The conducted treadmill training program was capable of increasing the femoral neck BMD more than the core training program in postmenopausal osteoporotic women, while both programs had no effect on improving the lumbar spine BMD.

\section{ACKNOWLEDGEMENTS}

The authors appreciate the great effort exerted by all the participants who kindly participated in the study.

\section{ABBREVIATIONS}

BMD - Bone Mass Densities

DEXA - Dual energy $x$-ray absorptiometry

\section{Conflicts of interest: None}

\section{REFERENCES}

[1]. Zehnacker C, and Dougherty A. Effect of weighted exercise on bone mineral density in postmenopausal women. A systematic review. Journal of Geriatric Physical Therapy. 2007;30(2):79-88.

[2]. Bonaiuti D, Shea B, lovine R, Negrini S, Robinson V, Kemper HC, Wells G, Tugwell P, Cranney A. Exercise for preventing and treating osteoporosis in postmenopausal women. Cochrane Database Syst Rev. 2002;(3):CD000333.

[3]. Stengel SV, Kemmler W, Lauber D, Kalendar WA, Engelike K. Differential effects of strength versus power training on bone mineral density in postmenopausal women: A 2 year longitudinal study. $\mathrm{Br}$ J Sports Med. 2007;41(12):649-655.

[4]. Hall J, Smidt G, O'Dwyer K, and Lin S. Relationship between trunk muscle torque and bone mineral content of the lumbar spine and hip in healthy postmenopausal women. Physical Therapy. 1990;70:690699.

[5]. Gari M. Bone Mineral Status Response to Treadmill Walking Exercise in Postmenopausal Women. AmEuras J Sci Res. 2009;4(4):246-249.

[6]. Fredericson $M$, and Moore T. Muscular balance, core stability, and injury prevention for middle- and long-distance runners. Phys Med Rehabil Clin N Am. 2005;16(3):669-689. 
[7]. Zimmermann C, Smidt G, Brooks J, Kinsey W, Eekhoff T. Relationship of extremity muscle torque and bone mineral density in postmenopausal women. Physical Therapy. 1990;70:302-309.

[8]. Kohrt W, Ehsani A, and Bigre S. Effects of exercise involving predominantly either joint reaction or ground reaction forces on bone mineral density in older women. J Bone Min Res. 1997;12(8):12531261.

[9]. Howe TE, Shea B, Dawson LJ, Downie F, Murray A, Ross C, Harbour RT, Caldwell LM, Creed G. Exercise for preventing and treating osteoporosis in postmenopausal women. Cochrane Database Syst Rev. 2011;6(7):CD000333.

[10]. Franck H, Munz M, and Scherrer M. Evaluation of dual-energy $X$ ray absorptiometry bone mineral measurement-comparison of a single-beam and fan-beam design; The effect of osteophytic calcification on spine bone mineral density. Calcif Tissue Int. 1995;56(3):192-195.

[11]. Lekamwasam S, and Lenora R. Effect of leg rotation on hip bone mineral density measurements. J Clin Densitom. 2003;6(4):331-336.

[12]. McGill S. Low Back Disorders: Evidence based prevention and rehabilitation, 2nd ed. Champaign, IIlinois, USA: Human Kinetics, pp. 216-222. 2002.

[13]. McGill S. Low back disorders: evidence-based prevention and rehabilitation, 2 nd ed. Champaign, IL, USA: Human Kinetics, pp. 221-229, 2007.

[14]. Franks K, Brown L, Coburn J, Kersey R, and Bottaro M. Effects of Motorized vs Non-Motorized Treadmill Training on Hamstring/Quadriceps Strength Ratios. J Sports Sci Med. 2012;11(1):71-76.

[15]. Nikander R, Sievänen H, Heinonen A, Daly R, UusiRasi $K$, and Kannus P. Targeted exercise against osteoporosis: A systematic review and meta-analysis for optimising bone strength throughout life. BMC Med. 2010;8:47.

[16]. Schmitt MN, Schmitt J, and Dören M. The role of physical activity in the prevention of osteoprosis in postmenopausal women-An update. Maturitas. 2009;63:34-38.
[17]. Habibzadeh N, Effects of two-month walking exercise on bone mass density in young, thin women. Biomedical Human Kinetics. 2010;2:5-8. DOI: 10.2478/v10101-010-0002-1.

[18]. Nelson M, Fiatarone M, Morganti C, Trice I, Greenberg R, and Evans W. Effects of High-Intensity Strength Training On Multiple Risk Factors for Osteoporotic Fractures. J Am Med Assoc. 1994;272:1909-1914.

[19]. Chien MY, Wu YT, Hsu AT, Yang RS, Lai JS. Efficacy of a 24-week aerobic exercise program for osteopenic postmenopausal women. Calcif Tissue Int. 2000;67(6):443-8.

[20]. Kerr D, Morton A, Dick I, Prince R. Exercise effects on bone mass in postmenopausal women are site specific and load-dependent. J Bone Miner Res. 1996;11:218-225.

[21]. Bemben DA. Exercise interventions for osteoporosis prevention in postmenopausal women. J Okla State Med Assoc. 1999;92:66-70.

[22]. Korpelainen R, Keinänen-Kiukaanniemi S, Heikkinen J, Väänänen K, Korpelainen J. Effect of impact exercise on bone mineral density in elderly women with low BMD: a population-based randomized controlled 30-month intervention. Osteoporosis International. 2006;17(1):109-118.

[23]. Bergstrom I, Landgren B, Brinck J, and Freyschuss B. Physical training preserves bone mineral density in postmenopausal women with forearm fractures and low bone mineral density. Osteoporos Int. 2008;19(2):177-83.

[24].Shanb AA, and Youssef EF. The impact of adding weight-bearing exercise versus nonweight bearing programs to the medical treatment of elderly patients with osteoporosis. J Family Community Med. 2014;21(3):176-181. Doi: 10.4103/2230-8229. 142972

[25]. Lester E, Mark L, Urso Maria Urso K, Evans Rachel R. Pierce Joseph, A. Spiering Barry, M. Maresh Carl et al., Influence of exercise mode and osteogenic index on bone biomarker responses during short term. Bone. 2009;45:768-776.

How to cite this article:

Mohamed Mostafa M. Essa, Hossam El-Din Fawaz, Hamada Ahmed Hamada, Mariam Abd-Elmoneem Ameer, Salam Mohamed Elhafez. EFFECT OF CORE AND TREADMILL TRAININGS ON SKELETAL MINERALIZATION IN POST-MENOPAUSAL OSTEOPOROTIC WOMEN. Int J Physiother Res 2017;5(5):2349-2355. DOI: 10.16965/ijpr.2017.209 